\title{
Aprendizaje del inglés por medio de plataformas virtuales en el aula
}

\section{Learning English through virtual platforms in the classroom}

Cynthia Enríquez Luna ${ }^{1}$

\section{Resumen}

Recientemente, las plataformas virtuales se han incorporado como base de las estrategias de enseñanza que utilizan los docentes en el aula, pues han demostrado su impacto positivo en el proceso de aprendizaje de los estudiantes. Una de las razones más importantes para diseñar clases utilizando la tecnología es que los estudiantes puedan aprender y, al mismo tiempo, tener una experiencia interesante con el conocimiento, en la cual están compartiendo, generando y desarrollando habilidades significativas para la vida. Esta propuesta didáctica se enfoca en demostrar que como maestros, podemos planificar nuestras clases de una manera diferente, donde el estudiante es el centro de su proceso de aprendizaje, para desarrollar diferentes habilidades en una experiencia divertida y significativa, utilizando las Tecnologías de la Información en toda una fase de enseñanza-aprendizaje.

\section{Palabras clave}

Aprendizaje del inglés, estudiantes, gamificación, Tecnologías de la Información y de la Comunicación.

\footnotetext{
Abstract

Recently, teachers have been incorporating virtual platforms in their classes as teacher techniques and strategies, because it has proved a positive impact on the learning process of the students. One of the most important reasons for us as teacher to design classes using the technology is that students can

${ }^{1}$ Cynthia Enríquez Luna. Docente de la Facultad de Organización Deportiva de la Universidad Autónoma de Nuevo León, México. Se desempeña también en el nivel medio superior en la Preparatoria 7 "Dr. Oscar Vela Cantú". Sus áreas de interés son: aprendizaje por medio de las TIC, gamificación y trabajo colaborativo. Correo electrónico: cyntienriquez@hotmail.com

ID: http://orcid.org/0000-0002-1497-8638
} 
learn and at the same time, have an interesting experience with knowledge, in which they are sharing, generating and building skills for life. This didactic proposal is focus on demonstrating that we as teachers can plan our classes in a different way, where the student is the center of his/her learning process, to develop different skills in a fun and significant experience through the application of the ICTs.

\section{Keywords}

English learning, students, gamification, Information and Communication Technologies.

\section{Introducción}

Con los cambios que han traído consigo la sociedad de la información y comunicación, y adicionalmente, las tecnologías de la información y de la comunicación, se ha visto un impacto positivo para poder introducir prácticas innovadoras en el aula, y se reconoce la necesidad de lograr un conocimiento adecuado del uso y aplicación de las TICs en las clases, como una fuente de aprendizaje autónomo por parte de los estudiantes. La enseñanza y el aprendizaje electrónico, en este contexto, se han convertido en una necesidad a tomar en cuenta, para la construcción de nuevos ambientes de aprendizaje.

En este trabajo, se presentan las ventajas del aprendizaje del inglés, y se presenta una propuesta basada en el uso de plataformas virtuales como base para el desarrollo de competencias específicas, y la manera en cómo se apropia el estudiante de las estructuras gramaticales desde una perspectiva diferente.

Como resultado de lo anterior surge la siguiente propuesta, en la cual se propone la implementación de la gamificación en el aula, para la dinamización de las prácticas educativas, a la vez que se fomenta el trabajo en equipo por medio del consenso y la aplicación de las estrategias, y por último, se obtiene una retroalimentación del proceso, al mismo tiempo que se ve una motivación intrínseca por lograr un objetivo que coadyuva al fomento de un ambiente positivo de aprendizaje en el que se comparten ideas entre los mismos protagonistas del proceso de aprendizaje. Se plantea una secuencia didáctica, siguiendo una metodología general en la enseñanza: Fase de Inicio, Desarrollo y Cierre, a seguir con sus recursos específicos cuya finalidad es favorecer el aprendizaje del inglés en los estudiantes que cursan el Nivel Medio Superior utilizando las tecnologías de la información. 


\section{Justificación de la Propuesta}

Hoy en día, el aprendizaje del inglés es cada vez más necesario, pues el contexto laboral y globalizador que nos envuelven, exigen un dominio de un segundo idioma. En este contexto, el aprendizaje de una segunda lengua surge como una demanda de la sociedad, como una competencia para la vida y la formación integral de los estudiantes que les permitirá ampliar su visión de otras culturas, lograr un mejor rendimiento académico y a la vez, poderse comunicar en una segunda lengua. Esta propuesta pretende mostrar que la educación presencial, en este caso referido al aprendizaje del inglés, puede llegar a evolucionar hacia un nuevo paradigma para la formación de las nuevas generaciones, al enriquecer sus procesos de enseñanza y de aprendizaje con la incorporación de las TIC para apoyar el desarrollo de competencias.

\section{Contexto de aplicación}

El contexto específico de la Preparatoria No. 7, Puentes se encuentra ubicado un medio urbano en Ave. Las Puentes y Sierra de Santa Clara, Las Puentes 1er Sector, 66460 San Nicolás de los Garza, N.L. La escuela preparatoria cuenta con 34 aulas equipadas cada una con un pizarrón electrónico, un proyector y una computadora, la cual cuenta con acceso a internet como una herramienta disponible para docentes y alumnos, todo esto como recursos tecnológicos de apoyo para las clases. Cada una de estas aulas cuenta además de un pizarrón blanco, sus respectivos mesa bancos, un escritorio, y un clima, todos estos elementos del mobiliario que en conjunto acondicionan y establecen los parámetros de la organización del ambiente de aprendizaje en el aula de cada una de ellas. En referencia al nivel socioeconómico de los estudiantes, se puede decir que cuentan con un teléfono celular cada uno, y en su mayoría tiene acceso a internet, lo que propicia la utilización de la tecnología en el aula. Por último, en lo referente al contenido, la propuesta se enfoca en la unidad de aprendizaje de inglés IV, referido al contenido conceptual del Present Perfect

\section{Fundamentación Teórica}

Enseñanza y Aprendizaje

Al hablar de una propuesta didáctica, es importante situar al lector en una perspectiva específica de lo que se entiende por enseñanza, pues este enfoque permitirá establecer un punto de partida sobre los roles que se involucran en el proceso de enseñanza-aprendizaje y las acciones que llevan a cabo cada uno de los actores. 
De acuerdo con José Gimeno Sacristán y Ángel I. Pérez Gómez (1992), la vida del aula es concebida como un conjunto de intercambios socioculturales, en donde hay una reciproca influencia en la relación alumno profesor, se enfatiza en el individuo y la relaciones de intercambio de significados que surgen en el proceso. Por esto, es importante tener en cuenta la ideología del docente frente a la significación de su propia práctica y a la manera en que concibe el aprendizaje, pues de esto dependerán las relaciones que se originen en el proceso.

Según Heidegger (1971), el acto de enseñanza es más complejo que el del aprendizaje, pues enseñar es dejar que el otro aprenda. De este modo, no se aprende del maestro sino a través de él. A través de la enseñanza es que se da a conocer una experiencia, un contenido específico o una habilidad que se pretende que ellos desarrollen después, por esto es que el docente establecerá las condiciones del contexto, y diseñará estrategias en el acto educativo para que los alumnos se apropien de ellos.

Ahora bien, Piaget (2001) refiere que enseñar es permitir que el niño descubra y cree por sí mismo; generar situaciones en las que él pueda movilizar sus estructuras mentales. Así pues, la enseñanza hace parte del día a día. No es una práctica exclusiva del docente o profesor, como se cree.

Es un saber natural o particular que se da a conocer por la capacidad y la cualidad del que lo enseña.

Por otro lado, el aprendizaje como una actividad cognitiva constructiva supone: a) el establecimiento de un propósito: aprender; y b) una secuencia de acciones orientadas a alcanzar o satisfacer este propósito. Por lo tanto, el aprendizaje es un proceso en el cual el sujeto se relaciona con el objeto a través de la conexión, acción, acomodación e incorporación de la información. (Flores, 2013)

En correspondencia a lo anterior, es importante establecer que se entiende entonces por un aprendizaje significativo, pues esto nos dará la pauta para diseñar condiciones en las que se propicie el mismo:

El aprendizaje significativo es el proceso según el cual se relaciona un nuevo conocimiento o información con la estructura cognitiva del que aprende de forma no arbitraria y sustantiva o no literal. Esa interacción con la estructura cognitiva no se produce considerándola como un todo, sino con aspectos relevantes presentes en la misma, que reciben el nombre de ideas de anclaje (Ausubel, 1976, 2002; Moreira, 1997).

De esta manera, para que el aprendizaje sea significativo se debe aludir a los conocimientos previos del estudiante $\mathrm{y}$ en combinación con los 
conocimientos adquiridos crear nuevos conocimientos y aplicarlos al ámbito de actuación generando motivación.

En consecuencia, es de gran relevancia que el docente incorpore propuestas innovadoras en el aula que le permitan enriquecer y potenciar aprendizajes significativos en los estudiantes, bajo la premisa de que para llevarlo a cabo tendrá que disponer de estrategias específicas en el contexto de aprendizaje, entendiendo una estrategia como un procedimiento organizado, formalizado y orientado a la obtención de una meta claramente establecida. Su aplicación en la práctica diaria requiere del perfeccionamiento de procedimientos y de técnicas cuya elección detallada y diseño son responsabilidad del docente (Tecnológico de Monterrey, 2010).

En este trabajo, se retomarán las percepciones anteriores para enunciar una propuesta didáctica que toma en cuenta el rol activo del docente para diseñar diferentes espacios en los que el que aprende pueda construir aprendizajes significativos, específicamente en el aprendizaje de una segunda lengua, el cual, además de ser un proceso intelectual, pone a los estudiantes en contacto con sus iguales en formas nuevas y significativas y comienza su adquisición de perspectivas más amplias y más socializadas, creándoles, al mismo tiempo, nuevas necesidades y problemas.

\section{Importancia del Inglés en el Siglo XXI}

Hoy en día, el aprendizaje del inglés es cada vez más necesario, pues el contexto laboral y globalizador que nos envuelven, exigen un dominio de un segundo idioma. En este contexto, el caso de México, el cual se encuentra en medio de las Reformas educativas, tiene un pendiente por resolver respecto al aprendizaje de una segunda lengua, pues a pesar de que se ha reconocido la importancia de aprender inglés y las ventajas que tiene para los estudiantes en su desarrollo académico, no ha tenido gran avance en los indicadores tanto mundiales.

En el último estudio que realizó el EF English Proficiency Index (EF EPI), México, con un puntaje de 51.57, quedó en el lugar 44 en el mundo (EPI2017), lo que evidencia la importancia de atender la importancia que tiene el inglés para nuestros estudiantes.

A partir de lo anterior, se puede decir que existen numerosos estudios sobre como el bilingüismo repercute de manera positiva en el desarrollo general del proceso de enseñanza-aprendizaje. Madrid (2006), establece una serie de argumentos a favor de su implantación en el sistema educativo basados en estudios experimentales de otros expertos que podríamos concretar en: el aprendizaje de dos lenguas conlleva ventajas cognitivas y lingüísticas para el alumnado. Existe una relación positiva entre las lenguas 
que se estudian, que facilita la transferencia entre ellas y el aprendizaje de los aspectos académicos y conceptuales (Cummins, 1999). La educación bilingüe tiene un efecto positivo en el rendimiento académico (Greene, 1998). Asimismo otros autores citados en dicho estudio nos dicen que la educación lingüística es más eficaz cuando tiene lugar en situaciones auténticas; y cuando las asignaturas que se enseñan interesan a los estudiantes, su motivación aumenta y ello favorece la adquisición de la segunda lengua (Riagáin y Lüdi, 2003).

Por lo anterior, es cada vez más necesario que los docentes que imparten esa unidad de aprendizaje en los niveles obligatorios, ofrezcan una experiencia de aprendizaje innovadora a los estudiantes.

\section{Uso de las tecnologías en el aula}

En lo que concierne a la enseñanza del inglés como lengua extranjera, las tecnologías de la información y la comunicación (TIC) han sido de gran ayuda para mediatizar los procesos. El uso de blogs, wikis, páginas web, plataformas virtuales, entre otros, han propiciado tanto en el docente como al estudiante nuevas formas de potenciar las habilidades comunicativas. Por esto, las instituciones que imparten educación se ven en la obligación de enseñar inglés y desarrollar habilidades lingüísticas por medio de las TIC.

De acuerdo al Informe Horizont, Edición 2008, con la incorporación de las Tecnologías de la Información en el aula, se plantean uno retos críticos para la educación, los que se presentan a continuación de forma textual. Se plantean como la evidencia de los cambios que se han generado en el mundo con respecto a la forma como accedemos a la información, como nos comunicamos y en general como nos impactan las nuevas tecnologías en nuestras vidas: Los cambios significativos en la enseñanza, la docencia, la expresión creativa y el aprendizaje han generado una necesidad de innovación y liderazgo en todos los niveles de la academia, el énfasis renovado en el aprendizaje en colaboración empuja a la comunidad educativa a desarrollar nuevas formas de interacción y evaluación, el mundo académico se enfrenta a la necesidad de encontrar modos de crear contenidos valiosos con las herramientas actuales (Correa, s/f).

En este punto, es importante mencionar que si se quiere incorporar, como docente, las Tecnologías de la información que el estudiante es protagonista activo de su aprendizaje y que el aprendizaje es personal y social (Flores, 2013).

En referencia a lo anterior, a continuación se enuncia una estrategia innovadora que fundamenta la propuesta didáctica planteada para este 
trabajo, en donde la premisa principal es el uso de las Tecnologías de la Información en el aula, en este caso, por medio de Herramientas de Respuesta de Audiencia.

\section{Gamificación en el aprendizaje.}

Las actividades interactivas se entienden como aquellas acciones que se relacionan directamente con otras que han acontecido previamente. El Gamification se refiere a la utilización de mecánicas de juego como la inmersión (multimedia), motivación, compromiso, concentración, esfuerzo y reto en el diseño de actividades de aprendizaje. (Gallego, 2016). Su objetivo principal es la influencia en el comportamiento de las personas, independientemente de otros objetivos secundarios como el hecho que las personas disfruten durante la realización del juego. Además, genera experiencias, origina sentimientos de autonomía y de influencia en las personas, produciendo un cambio notable en el comportamiento en éstas.

Kahoot proporciona dos plataformas, para alumno y profesor, y el cuestionario diseñado puede incluir imágenes y vídeos. Cada vez que el profesor decide activar un cuestionario se habilita una sesión con un identificador. Este identificador es utilizado por los alumnos para conectarse a la actividad. A cada pregunta se le asigna un tiempo máximo de respuesta. Una vez que termina este tiempo ya no se permiten más contestaciones. Después de cada cuestión muestra la puntuación de los 5 mejores estudiantes. Esta puntuación está basada en el acierto de cada pregunta y en la rapidez de respuesta, es decir, dos alumnos que responden correctamente tienen puntuaciones diferentes dependiendo de lo rápida que haya sido la respuesta.

Por otro lado, Socrative es una plataforma virtual que permite tanto a profesores como a alumnos conectarse a través de sus dispositivos móviles (celular, tableta o computador portátil) estando en clase. Esta plataforma es de acceso gratuito y cada profesor puede crear su propia cuenta. Fue inventada por Berté. West u Duncan (2014), y actualmente la administra Mastery Connect. El objetivo de esta plataforma es incentivar al alumno a participar en clase respondiendo las preguntas a través de su dispositivo móvil. Esto quiere decir que dentro de la clase los alumnos se conectan a socrative.com y responden las preguntas de una actividad propuesta por el profesor. Mientras, el docente es capaz de ver el progreso individual y grupal del grupo curso en tiempo real. 
Retomando lo anterior, es importante mencionar que la implementación de determinada aplicación dependerá del el fin que se persiga en el proceso de instrucción.

\section{Secuencia didáctica y su diseño}

En el marco actual de la enseñanza, la introducción de actividades diversas de aprendizaje, y de instrumentos innovadores de evaluación constituyen un factor clave que ayuda a dinamizar este nuevo modelo formativo, cuyo eje central lo conforman el proceso de aprendizaje del estudiante y el papel que desempeñan los docentes, quienes han de asumir este nuevo reto para centrar sus esfuerzos en facilitar el logro de las competencias y estimular el aprendizaje del alumnado.

De acuerdo con este precepto, en este espacio se expondrá una secuencia didáctica enfocada a potenciar la adquisición de un contenido conceptual en específico, el cual es el Present Perfect Tense, pasando por las tres fases de la enseñanza: fase de inicio, desarrollo y cierre, utilizando diversas aplicaciones tecnológicas en el aula como Socrative y Kahoot, en tiempo real. Lo anterior, permitirá enriquecer los conocimientos de los alumnos, desde una perspectiva diferente de la enseñanza, en donde el objetivo final es trasladar esos conocimientos a los contextos y situaciones reales de los que aprenden.

En la figura 1, se presentan los datos generales de la Unidad de Aprendizaje, así como los contenidos conceptuales y procedimentales a abordar en la propuesta. 
Figura 1

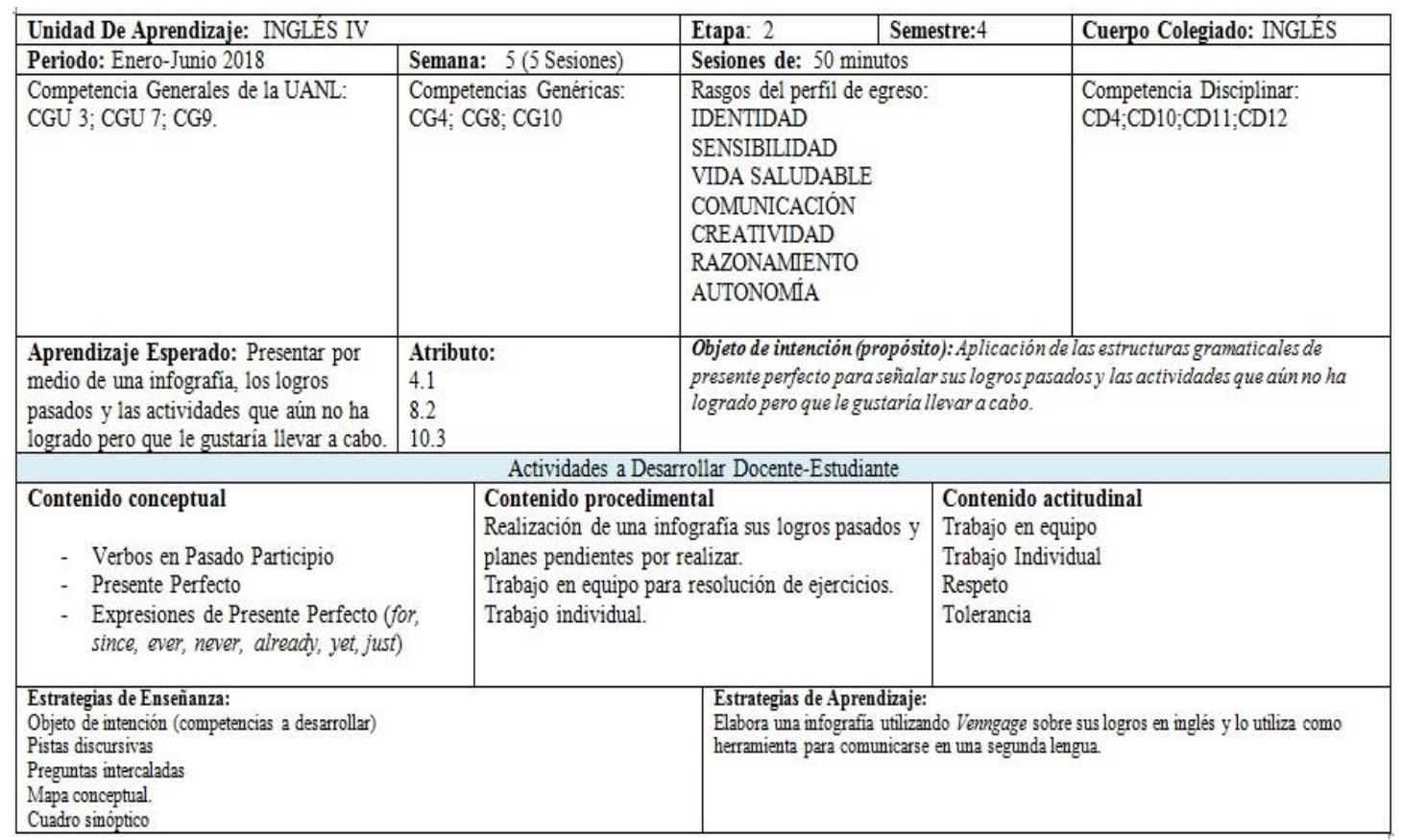

Algunos de los objetivos que se pretenden lograr con la propuesta son:

1. Diagnosticar conocimientos previos a través de un crucigrama de los verbos en pasado participio utilizando una actividad interactiva en línea. Figura 2.

https://www.englisch-

hilfen.de/en/exercises/irregular verbs/crossword 6 7.htm

2. Identificar verbos en pasado participio utilizando los ejercicios práctica. Figura 3.

https://web2.uvcs.uvic.ca/elc/studyzone/410/grammar/pperf2.htm Reconocer estructuras del pasado participio por medio de la plataforma GoConqr. Figura 4. https://www.goconqr.com/en/p/13079194

3. Utilizar el uso del Presente Perfecto con for,since para describir por cuanto tiempo han realizado alguna actividad, reforzando contenidos con una actividad en Kahoot.Figura 5

4. Aplicar la estructura del presente perfecto con sus palabras clave: ever, never, already, just, yet, para realizar una infografía, resolución de ejercicios en página en línea. Figura 6 https://www.grammarbank.com/ever-never-just-already-yet.html

5. Presentación y publicación de infografía en plenaria con los logros y experiencias llevadas a cabo, y las actividades pendientes por realizar; 
RECIE. Revista Electrónica Científica de Investigación Educativa

Vol. 4, núm. 2, enero-diciembre 2019, pp. 1209-1221.

utilizando las estructuras del Presente Perfecto utilizando la plataforma Plataforma Venngage. $\quad$ Figura 7. https://infograph.venngage.com/onboarding\#

Figura 2

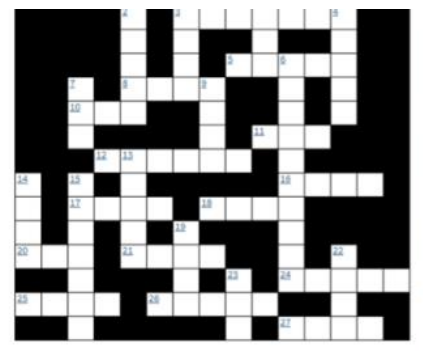

2. Simple Putcolasiva

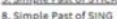

10. simple Pant of EAT

11. Simpie Part offere

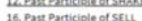

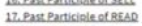

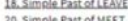

21. Simnde Fartot opaw

26. Simple Part of Dann:

25.Simple Farted fiY

27. Pant Particiole of Maxs

Down

2.Simple partot choos

2. Past Particiale of as

4 . Past Particiole eft Taks

Figura 4

Figura 5

Present Perfect 2 Irregular Past Participles

To make the present perfect, you need to use HAVE + PAST PARTIIPIE, The past participles of most verbs are formed using - ED, but some common verbs have unusual
past participles. In this exercise, you can test your knowledge of irregular past participles. click on the button beside the correct answet.

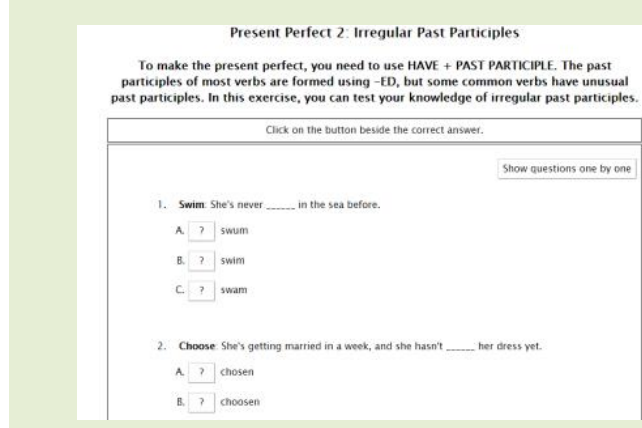

Figura 3

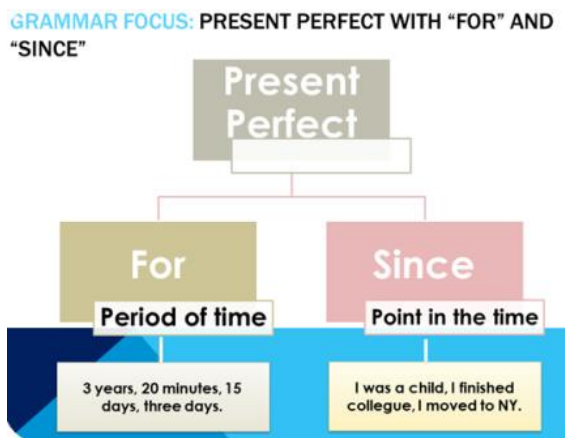

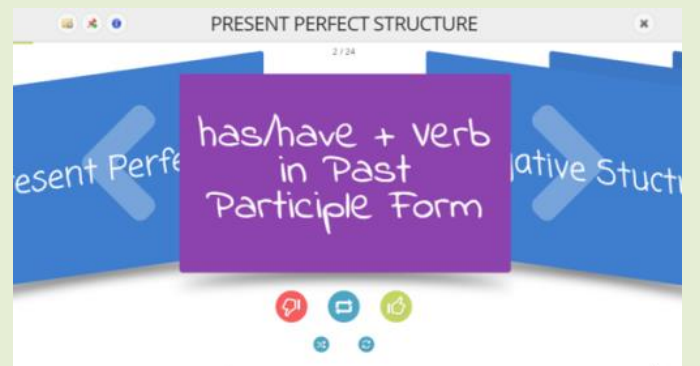



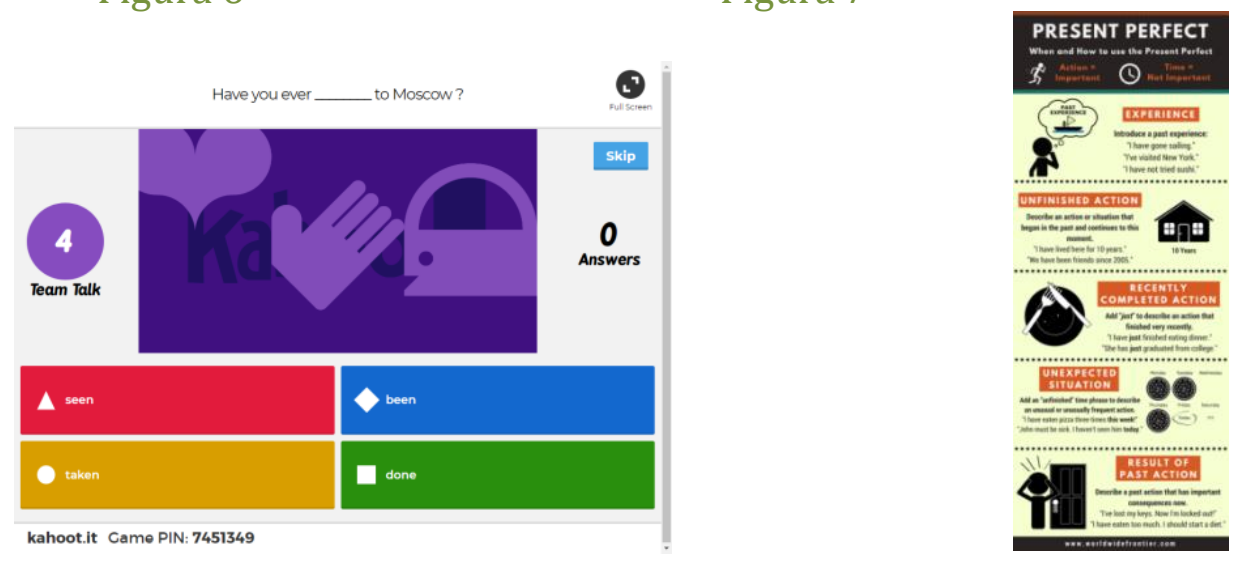

\section{Comentarios Finales}

La presente propuesta ha sido tomada como referencia al aprendizaje del inglés, para esto se tomó como referencia la enseñanza del Present Perfect para evidenciar su aplicación, pero la aplicación de la misma puede ser precedente de diversos contenidos, lo que habría que destacar para otros contenidos conceptuales, es su naturaleza misma. De este modo son los objetivos didácticos como el modo de aprendizaje, los que determinan el método y la aplicabilidad de la propuesta a otros escenarios con otros contenidos.

Por otro lado, el reto para los docenes, consiste en la definición de una metodología, vista desde un enfoque pedagógico, que oriente y posibilite la construcción del conocimiento. En este sentido, los diferentes actores del sistema educativo debemos replantear de forma constante el papel que desempeñamos en el sistema, dado que nuestros métodos y estrategias deben evolucionar a la par con el mundo cambiante y al mismo tiempo, convertirlos en factor nuevos de cambios e innovaciones, por esta razón, la incorporación de las tecnologías de la información con una planeación sistemática y bien orientada a los objetivos propuestos, permitirá potenciar el aprendizaje de las nuevas generaciones. Por último, la gradualidad de la dificultad de las actividades plantea retos a los estudiantes, por medio del uso de la tecnología, en pares, en equipo, de manera individual; todo lo anterior para lograr una apertura en la que el alumno logre una interiorización de las estructuras y de los usos adecuados a la gramática.

Finalmente, se reconoce como la tecnología funge como elemento mediador en el proceso de enseñanza aprendizaje, pues es necesario el 
RECIE. Revista Electrónica Científica de Investigación Educativa Vol. 4, núm. 2, enero-diciembre 2019, pp. 1209-1221.

docente reconozca las TIC como un medio y las aplique en su quehacer diario, en donde de éste y del estudiante depende sacarle el mayor provecho.

\section{Referencias}

Ausubel, D. (1976). Psicología educativa. Un punto de vista cognoscitivo. Ed. Trillas. México.

Ausubel, D. (2002). Adquisición y retención del conocimiento. Una perspectiva cognitiva. Ed. Paidós. Barcelona.

Bruner, J. (1982). La importancia de la educación. Barcelona: Paidós Educador.

Correa, F. (s/f). Ambientes de aprendizaje en el siglo XXI. Línea I + D en Informática Educativa; Universidad EAFIT. Archivo PDF recuperado el 15 de enero de 2018.

Cummins, J. (1999). Research, Ethics and Public Discourse: The Debate on Bilingual Education. Presentation at the National Conference of the America Association of Higher Education. Washington, D.C.

Flores, I. (2013). Ambientes de aprendizaje y el trabajo docente en entornos de aprender a aprender. En Flores-Alanís, I.M.; González-Salinas, A.; y Meza-Mejía, M. del C. (2013). De Aprendiz a Aprendiente, Monterrey, Facultad de Filosofía y Letras, Universidad Autónoma de Nuevo León. Gallego, G. (2016). Ambientes electrónicos de aprendizaje en la era digital. Universidad Autónoma de Occidente. Libro electrónico, primera edición ISBN Digital: 978-98-8713-92- 2.

Greene, J. (1998). A meta-analysis of the Effectiveness of Bilingual Education. Bilingual Research Journal 21 (2,3): 103-122.

Heidegger, M. (1971). Was heisst denken? Tubinga (Alemania): Max Niemeyer.

Howatt, A. (1984). A history of English Language Teaching. Oxford: Oxford University Press.

Kapp. K. (2012). The Gamification of Learning and Instruction: Game Based Methods and Strategies for Training and Education. San Francisco, Pfeiffer, May.

Moreira, M. (1997). Aprendizagem Significativa: um conceito subyacente. En M.A. Moreira, C. Caballero Sahelices y M.L. Rodríguez Palmero, Eds. Actas del II Encuentro Internacional sobre Aprendizaje Significativo. Servicio de Publicaciones. Universidad de Burgos. Págs. 19-44.

Riagáin, P.; y Lüdi, G. (2003). Bilingual Education: some Policy Issues. Strasbourg: Council of Europe 
Richards, J.; y Rodgers, T. (2001). Approaches and Methods in Language Teaching. (2nd. edition). USA: Cambridge University Press

Sacristán, J.; y Pérez, A. (1992). Comprender y transformar la enseñanza. Publicado por Ediciones Morata, Edición 10. ISBN 8471123738, 9788471123732. 441 páginas. 1992.

Tecnológico de Monterrey (2010) Qué son técnicas didácticas. Disponible en http://sitios.itesm.mx/va/dide2/tecnicas didacticas/quesontd.htm

Yong, E. y Bedoya, D. (s/f). De la educación tradicional a la educación mediada por TIC: Los procesos de enseñanza-aprendizaje en el siglo XXI. Archivo PDF recuperado el 31 de mayo de 2018. 
RECIE. Revista Electrónica Científica de Investigación Educativa Vol. 4, núm. 2, enero-diciembre 2019, pp. 1209-1221. 